\title{
Phenotypic Detection of Different Resistance Mechanism of Imipenem Resistance among Clinical Isolates of Enterobacteriaceae
}

\author{
M. Swetha*, K. Anuradha and Kiran Kavatagi \\ Department of Microbiology, Mysuru medical college and research institute, \\ Mysuru, Karnataka, India \\ *Corresponding author
}

\begin{abstract}
A B S T R A C T
Keywords

Carbapenemases, Modified Hodge

Test,

MBL,

AmpC.

\begin{tabular}{l}
\hline Article Info \\
\hline Accepted: \\
12 June 2016 \\
Available Online: \\
10 July 2016
\end{tabular}

Carbapenems are the mainstay in the management of infections by multi-drug resistant (MDR) Enterobacteriaceae. However, there are reports of increasing prevalence of carbapenem resistance in clinical isolates of Enterobacteriaceae mainly due to the production of metallo- $\beta$-carbapenemases (MBL), Klebsiella pneumoniae carbapenemase (KPC) and AmpC $\beta$-lactamases (AmpC). A simple and inexpensive testing method for screening of carbapenemase producers is essential. The aim of the present study is to determine phenotypically the presence of different resistance mechanism among imipenem resistant Enterobacteriaceae. The prospective study screened all Enterobacteriaceae isolated from blood, tracheal aspirate, urine and pus aspirates which were submitted to the microbiology laboratory. The isolates were screened according to susceptibility zone size $<23 \mathrm{~mm}$ of Imipenem on Kirby-Bauer disc diffusion method. The screened isolates were subjected to phenotypic assay Modified Hodge Test, combined Imipenem EDTA and Imipenem phenylboronic acid disc diffusion test, AmpC disc test were used for the detection of carbapenemases, MBL, KPC and AmpC $\beta$-lactamases, respectively. A total of 100 Imipenem resistant Enterobacteriaceae isolates comprising Klebsiella pneumoniae 37 (37\%); Escherichia coli 32 (32\%); Citrobacter freundii 7(7\%); Enterobacter sps 19(19\%);Proteus mirabilis 5(5\%) were included in the study. Out of the 100 isolates,39 (39\%) found positive for carbapenemases by modified Hodge test, 43 (43\%) were MBL producers by combined Imipenem EDTA disc diffusion test, 45 (45\%) were KPC producers by combined Imipenem phenylboronic acid disc diffusion test, 38 (38\%) were AmpC $\beta$ lactamase producers. KPC production was a predominant mechanism of carbapenem resistance among Klebsiella pneumoniae(67.6\%), MBL production in case of Escherichia coli( $65.6 \%)$, AmpC $\beta$-lactamases production among Enterobacter sps (63.2\%), Citrobacter freundii(71.4\%),Proteus mirabilis(60\%).Boronic acid-based inhibition testing for KPC detection and Combined Imipenem- EDTA disc test for detection of Metallo- $\beta$-lactamases (MBL) were found to be effective. Use of both MHT and combined imipenem EDTA and imipenem phenylboronic acid disc diffusion test as a screening method can increase the sensitivity of detection of carbapenemases. AmpC $\beta$-lactamase is also a contributory factor for carbapenem resistance among Enterobacteriaceae.
\end{abstract}




\section{Introduction}

Carbapenems are the mainstay in the management of infections by multi-drug resistant (MDR) Enterobacteriaceae. However, clinical utility of this group of antibiotic is under threat due to recent emergence and spread of Imipenem/ Meropenem resistant Enterobacteriaceae throughout the world (Binish Arif Sultan et al., 2013). The mainstay in carbapenem resistance is the production of enzyme carbapenemase that has been classified into four main classes (Ambler Class: A-D) according to their amino acid sequence, include class A carbapenemases (KPCtypes), class B or metallo-betalactamases (MBLs) (VIM, IPM, and NDM types), and class D oxacillinases (e.g.,OXA48-like enzymes) (Andrea Bartolini et al., 2014).

In addition, decreased susceptibility to carbapenems in Enterobacteriaceae may be caused by either extended spectrum betalactamases (ESBLs) or AmpC enzymes combined with drug decreased permeability, due to loss of porins (Andrea Bartolini et al., 2014). Owing to plasmid mediated mode of spread of metallocarbapenemase among Enterobacteriaceae, the risk of nosocomial infections, epidemic outbreaks and increased mortality is of great concern.

Therefore, an early, reliable and affordable laboratory method for the detection of carbapenemase-producing enterobacteriaceae is of utmost importance ${ }^{1}$ and also it is essential to detect and differentiate class A and B carbapenemases among Enterobacteriaceae isolates in the clinical laboratory that may provide substantial information before application of the more expensive molecular techniques. This study is focused on the simple phenotypic detection methods for the presence of different resistance mechanism among imipenem resistant Enterobacteriaceae.

\section{Materials and Methods}

A prospective study was conducted at the Department of Microbiology, MMC\&RI, MYSORE. A total of 100 Imipenem resistant Enterobacteriaceae isolated from various clinical specimens like sputum, pus, urine, blood etc were included in the study. These organisms were isolated from specimens like sputum, tracheal aspirate, pus, urine, blood, pleural fluid and ascitic fluid of patients admitted to different wards, which were sent to the microbiology laboratory for routine culture identification and sensitivity testing. All the Enterobacteriaceae isolates were screened for susceptibility zone size $<23 \mathrm{~mm}$ to Imipenem by Kirby-Bauer disc diffusion method according to CLSI guidelines (Clinical and Laboratory Standards Institute CLSI, 2012). The screened isolates having zone size $<23 \mathrm{~mm}$ considered Imipenem resistant were subjected to phenotypic assay Modified Hodge Test, combined Imipenem EDTA and Imipenem phenylboronic acid disc diffusion test and AmpC disc test were used for the detection of carbapenemases, MBL, KPC and AmpC $\beta$-lactamases, respectively.

Phenotypic detection of carbapenamase production by Modified Hodge test (MHT)

All the isolates were subjected to Modified Hodge test as per CLSI guidelines. A lawn culture of the 1:10 dilution of Escherichia coli ATCC 25922 as recommended by CLSI was done on Mueller Hinton agar plate and a $10 \mu \mathrm{g}$ meropenem susceptibility disk was placed in the centre of the test area. The test organism was then streaked in a straight line from the edge of the disk to the edge of the plate. Four strains were tested on the same 
plate with one disk and were incubated overnight at $35^{\circ} \mathrm{C} \pm 2{ }^{\circ} \mathrm{C}$ in ambient air for16- 24 hours.

Interpretation was done after 16- 24 hours of incubation. Positive Modified Hodge test showed a clover leaf-like indentation of the Escherichia coli 25922 strain growing along the test organism growth streak within the disk diffusion zone indicating production of carbapenemase and a negative test showed no growth of the Escherichia coli ATCC 25922 along the test organism growth streak within the disk diffusion.

\section{Combined Imipenem- EDTA disk test for detection of Metallo- -lactamases (MBL)}

All isolates were tested with the combined imipenem-EDTA test with appropriate positive and negative controls. Test organisms were inoculated on to Mueller Hinton agar plates as per CLSI guidelines. A $10 \mu \mathrm{g}$ imipenem disk and imipenem $(10 \mu \mathrm{g})$ EDTA (750 g) combined disk were placed on the plate (Hi- Media, Mumbai) and incubated for $16-18$ hours at $35^{\circ} \mathrm{C}$. The increase in inhibition zone with the imipenem and EDTA disk $7 \mathrm{~mm}$ than the imipenem disk alone was considered as a Metallo-Lactamase (MBL) positive strain.

\section{Use of Inhibitor for Differentiation of KPC}

Use of inhibitor phenylboronic acid (PBA) along with meropenem disc was used for detection of KPC.

The stock solution of PBA in the concentration of $20 \mathrm{mg} / \mathrm{ml}$ was prepared by dissolving PBA in DMSO. Twenty microliters (400 $\mu \mathrm{g}$ of PBA) from this solution was dispensed onto meropenem discs. The meropenem discs with inhibitor added was dried and used within $60 \mathrm{~min}$. On Mueller Hinton agar plate inoculated with test strain, two discs of meropenem were used. One disc of meropenem was without any inhibitor, one disc had PBA $(400 \mu \mathrm{g})$. The agar plates were incubated at $37^{\circ} \mathrm{C}$ overnight and the diameter of the growth inhibitory zone around these meropenem discs with inhibitor added was compared with that around the plain meropenem disc.

The isolate was considered KPC producing when the growth inhibitory zone diameter around the meropenem disc with PBA was increased $>5 \mathrm{~mm}$ compared with the growth-inhibitory zone diameter around the disc containing meropenem alone.

\section{Amp C disk test}

AmpC disk test was also done for the meropenem resistant strains for detection of AmpC $\beta$-lactamases5. On a MHA plate, lawn culture of $E$. coli ATCC 25922 was made from an overnight culture suspension adjusted to $0.5 \mathrm{McFarland}$ standard. A $30 \mu \mathrm{g}$ cefoxitin disk was kept on the surface of the agar. A blank disk $6 \mathrm{~mm}$ in diameter, Whatmann filter paper no.1 was moistened with sterile saline and inoculated with a few colonies of the test strain. The inoculated disk was then placed beside the cefoxitin disk almost touching it. The plate was incubated overnight at $37^{\circ} \mathrm{C}$. A flattening or indentation of the cefoxitin inhibition zone in the vicinity of the disk with test strain was interpreted as positive for the production of AmpC $\beta$-lactamase. An undistorted zone was considered as negative.

\section{Results and Discussion}

A total of 100 Imipenem resistant Enterobacteriaceae isolated from various clinical samples were studied. Out of 100 organisms, commonest isolates were Klebsiella pneumoniae 37\%, E.coli $32 \%$, Enterobacter spp 19\%, Citrobacter freundii 7\% and Proteus mirabilis 5\%. Among the 
100 Imipenem resistant isolates, 39 (39\%) found positive for carbapenemases by modified Hodge test, 43 (43\%) were MBL producers by combined Imipenem EDTA disc diffusion test, $45(45 \%)$ were KPC producers by combined Imipenem phenylboronic acid disc diffusion test, 38 $(38 \%)$ were AmpC $\beta$-lactamase producers.

KPC production was a predominant mechanism of carbapenem resistance among Klebsiella pneumoniae (67.6\%), MBL production in case of Escherichia coli (65.6\%), AmpC $\beta$-lactamases production among Enterobacter sps (63.2\%), Citrobacter freundii (71.4\%), Proteus mirabilis $(60 \%)$.

The emergence and spread of carbapenemresistant Enterobacteriaceae (CRE) producing acquired carbapenemases have created a global public health crisis. This massive spread, has forced routine analysis to elaborate reliable detection methods (Rai et al., 2014). In the present work we analyzed four different phenotypic tests for their ability to correctly identify carbapenem resistance mechanisms, to provide reliable and easy to set up workflow for the detection of carbapenemase and carbapenem hydrolyzing AmpC producers in clinical specimens.

In our study out of 100 imipenem resistant Enterobacteriaceae, Carbapenem resistance was witnessed at a higher rate in Klebsiella pneumoniae $37 \%$ when compared $32 \%$ in E. coli , $19 \%$ in Enterobacter sps , 7\% in Citrobacter freundii and 5\% in Proteus mirabilis. Wattal, et al., (2010) reported high prevalence of resistance to carbapenems ranging from 13 to $51 \%$ in $E$. coli and Klebsiella spp. Similar findings have been reported (Chauhan et al., 2015). In the present study out of 100 imipenem resistant Enterobacteriaceae, 39\% tested positive for production of carbapenemases by MHT and $61 \%$ isolates were negative.
Similar findings have been reported (Sathya Pandurangan et al., 2015). In this study, we have observed the occurrence of false results of carbapenemase production by the modified Hodge test (MHT) among isolates in which carbapenem reduced susceptibility or resistance was detected. It is suggested that the modified Hodge test should be used as a confirmatory test for carbapenemase production when the initial screening tests are indicative (Tsakris et al., 2010). However the main advantage of MHT is that different carbapenemase classes can be recognised in a single plate and apart from being technically demanding and time consuming, disadvantages of this test (MHT) include possible interpretation difficulties and cannot discriminate between various classes of carbapenemases and leads to false positives for Amp C and ESBL isolates (Lee et al., 2010).

In the present study three combined-disc tests employing imipenem alone and with PBA, EDTA, or both PBA and EDTA were tested for the differentiation of KPCs and MBLs among Enterobacteriaceae. It was found that out of 100 imipenem resistant Enterobacteriaceae, $43 \%$ of organisms found positive by MBL screen, $45 \%$ by KPC screen, both MHT and MBL were positive in $18 \%$ and both MBL and KPC were positive in $17 \%$. In a study conducted by Bansal et al. (2013) and Baraniak et al. (2013) they reported KPC producers were more common than the MBL producers, which is in concordance with our study. Authors from different parts of India have reported varying resistance rates of carbapenem in Enterobacteriaceae.

Currently MBLs and KPCs are considered a major threat in Enterobacteriaceae, representing a potential source of clinical failure in patients treated with almost all $\beta$ lactam regimens (Queenan et al., 2007). Although MBL and KPC 
carbapenemases are independently disseminated in different regions worldwide, (Queenan et al., 2007; Nordmann et al., 2009) their co-production has been reported in $K$. pneumoniae and currently is widely detected among Enterobacteriaceae. The production of both enzymes might contribute to their hydrolytic activity and levels of resistance to broad-spectrum $\beta$ lactams, as well as to the possible comigration of both enzymes. Moreover, KPC and MBL genes are often co-transferred with plasmid-mediated ESBL, fluoroquinolone and aminoglycoside resistance genes, (Beth et al., 1997) and this may possibly contribute to the dissemination of additional resistance mechanisms among MBL and/or KPC producers. Therefore, the accurate phenotypic detection of MBL and
KPC carbapenemases is important for clinical and epidemiological purposes, adding to studies on the preliminary characterization of the antimicrobial resistance mechanisms.

Among the 100 imipenem resistant isolates $82 \%$ isolates tested negative by both Methods(MHT and MBL), 83\% isolates tested negative by both Methods(KPC and MBL) in spite of being resistant to carbapenems by disk diffusion. The likely explanation could be over production of ESBL, or Amp C hyper producers with porin loss. Organisms that produce carbapenem-hydrolyzing enzymes frequently produce more than a single $\beta-$ lactamase.

Table.1 Distribution of organisms isolated among Enterobacteriaceae $(n=100)$

\begin{tabular}{|l|c|c|}
\hline \multicolumn{1}{|c|}{ Organism } & $\mathrm{N}$ & $\%$ \\
\hline Klebsiella pneumoniae & 37 & 37.0 \\
\hline E.coli & 32 & 32.0 \\
\hline Enterobacter sps & 19 & 19.0 \\
\hline Citrobacter freundii & 7 & 7.0 \\
\hline Proteus mirabilis & 5 & 5.0 \\
\hline
\end{tabular}

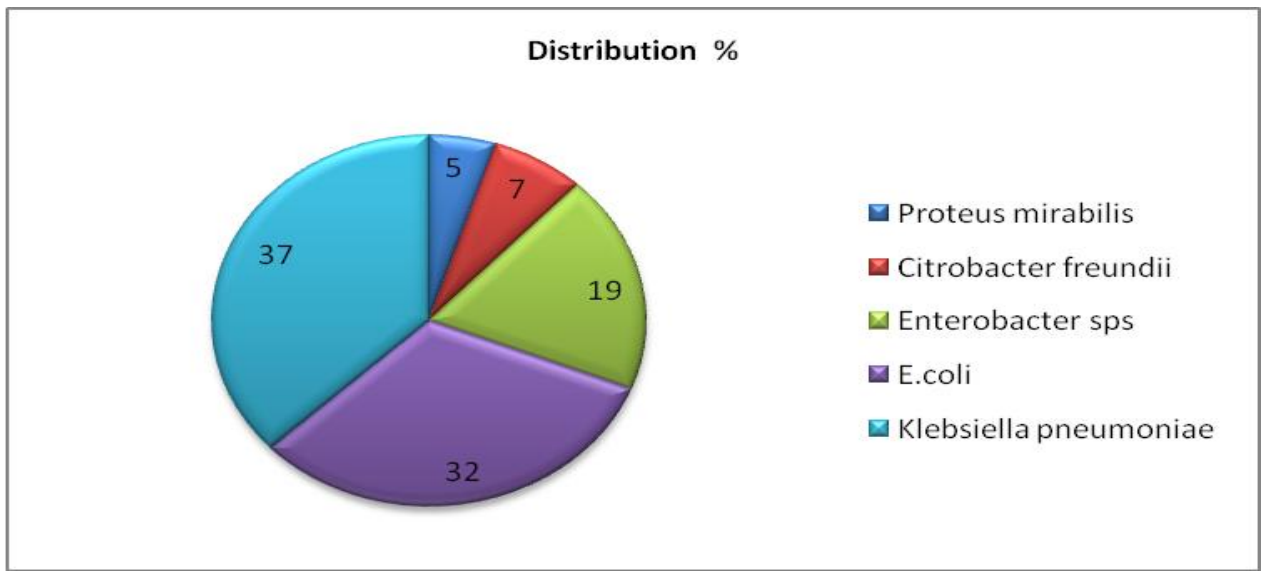


Table.2 Distribution of resistance mechanism among Enterobacteriaceae $(\mathrm{n}=100)$

\begin{tabular}{|l|c|c|}
\hline \multirow{2}{*}{} & \multicolumn{2}{|c|}{$\begin{array}{c}\text { Distribution of } \\
\text { Resistance } \\
\text { Mechanism among } \\
\text { Enterobactriacae }\end{array}$} \\
\cline { 2 - 3 } & $\mathrm{N}$ & $\%$ \\
\hline MHT & 39 & 39 \\
\hline MBL & 43 & 43 \\
\hline KPC & 45 & 45 \\
\hline AmpC & 38 & 38 \\
\hline MHT+MBL & 18 & 18 \\
\hline MBL+KPC & 17 & 17 \\
\hline
\end{tabular}

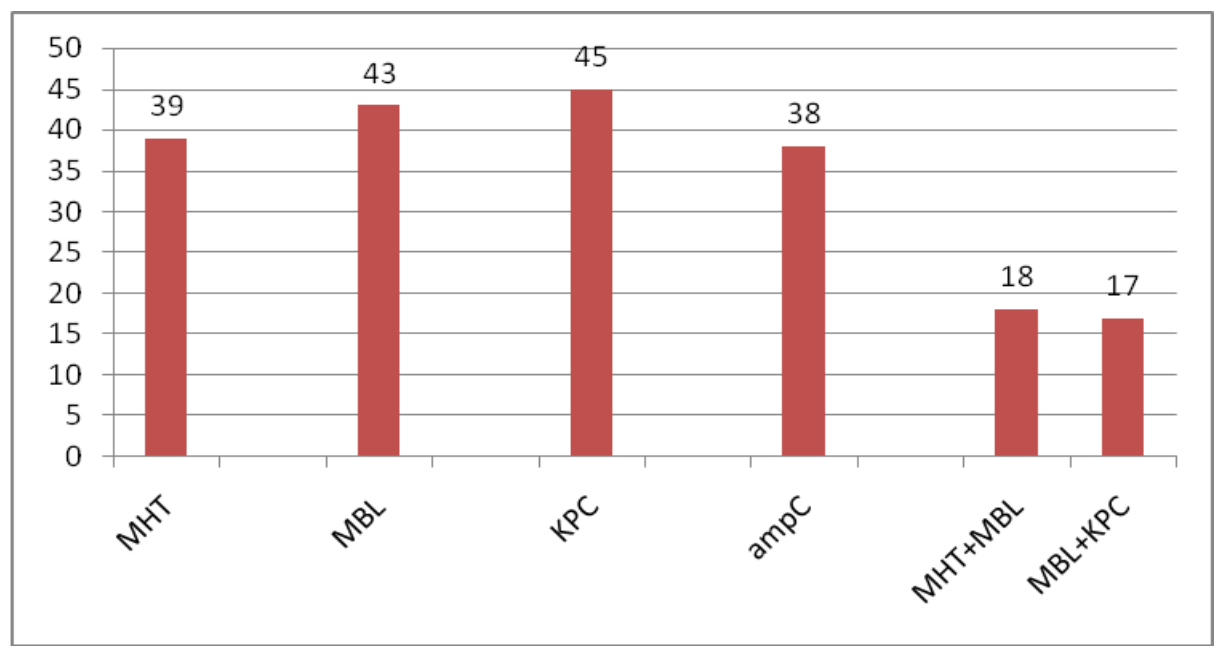

Table.3 Distribution of various types of $\beta$-lactamases in Family Enterobacteriaceae

\begin{tabular}{|c|c|c|c|c|c|c|c|c|}
\hline Organisms & $\begin{array}{l}\text { MHT } \\
\mathrm{n}(\%)\end{array}$ & $\begin{array}{l}\text { MBL } \\
\mathrm{n}(\%)\end{array}$ & $\begin{array}{l}\mathrm{KPC} \\
\mathrm{n}(\%)\end{array}$ & $\operatorname{ampCn}(\%)$ & $\begin{array}{l}\text { MHT+M } \\
\text { BL, n }(\%)\end{array}$ & $\begin{array}{l}\text { MBL+K } \\
\text { PC, } \mathrm{n}(\%)\end{array}$ & $\begin{array}{l}\text { MHT+MBL+ } \\
\text { KPC, n }(\%)\end{array}$ & $\begin{array}{l}\text { Carbapene } \\
\text { mase+ampC }\end{array}$ \\
\hline $\begin{array}{l}\text { Proteus mirabilis } \\
(n=5)\end{array}$ & $2(40 \%)$ & $1(20 \%)$ & $0(0 \%)$ & $3(60 \%)$ & $0(0 \%)$ & $0(0 \%)$ & $0(0 \%)$ & $1(20 \%)$ \\
\hline $\begin{array}{l}\text { Citrobacter freundii } \\
(n=7)\end{array}$ & $1(14.3 \%)$ & $1(14.3 \%)$ & $2(28.6 \%)$ & $5(71.4 \%)$ & $0(0 \%)$ & $0(0 \%)$ & $0(0 \%)$ & $2(28.6 \%)$ \\
\hline $\begin{array}{l}\text { Enterobacter sps } \\
(n=19)\end{array}$ & $5(26.3 \%)$ & $5(26.3 \%)$ & $8(42.1 \%)$ & $12(63.2 \%)$ & $2(10.5 \%)$ & $1(5.3 \%)$ & $0(0 \%)$ & $7(36.8 \%)$ \\
\hline $\operatorname{E.coli}(n=32)$ & $16(50 \%)$ & $21(65.6 \%)$ & $10(31.3 \%)$ & $9(28.1 \%)$ & $10(32.2 \%)$ & $6(18.7 \%)$ & $2(6.3 \%)$ & $9(28.1 \%)$ \\
\hline $\begin{array}{l}\text { Klebsiella } \\
\text { pneumoniae } \\
(n=47)\end{array}$ & $15(40.5 \%)$ & $15(40.5 \%)$ & $25(67.6 \%)$ & $9(24.3 \%)$ & $6(16.2 \%)$ & $10(27 \%)$ & $4(10.8)$ & $9(24.3 \%)$ \\
\hline
\end{tabular}




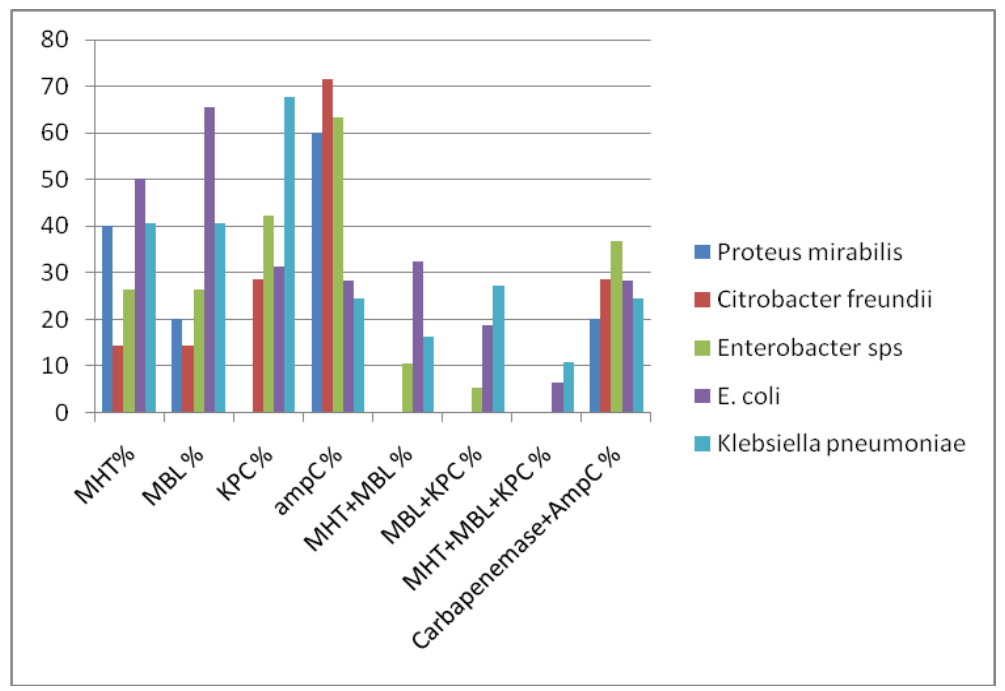

Amp C $\beta$-lactamase production was screened by AmpC disk test and found to be $38 \%$. A study done by Sathya Pandurangan et al., Amp C $\beta$-lactamase production was screened by cefoxitin disk diffusion and found to be $61 \%$. Moreover (AmpC, Ambler class C) a particular class of plasmidic cephalosporinases displays a slightly extended inducible hydrolytic activity towards carbapenems and therefore has to be taken into consideration during putative carbapenemase detection and subsequent antimicrobial treatment. We could not discriminate the different types of carbapenemases due to unavailability of PCR in our hospital and perhaps, this is the major limitation in our studies.

In conclusion, adequate detection of the production of carbapenemase in Enterobacteriaceae isolates is crucial for infection control measures and epidemiological analysis. In addition, it is important to make appropriate choice of anti-microbial therapy. Therefore, screening and confirmation of such isolates with simple and cost-effective methods is recommended in the absence of molecular techniques.

Based on the present study, the modified Hodge test for the routine phenotypic detection of carbapenemases, combined disk test using EDTA for MBL production and boronic acid disk test for KPC, can be employed in any laboratory settings where molecular diagnostic techniques are not available to detect this important mechanism of antimicrobial resistance.

Knowledge about the prevalence of CRE and other drug-resistant organisms in the intestine can help in formulating antibiotic policy in management of sepsis as a complication of extensive gut surgery or patients with haematological malignancy under chemotherapy or bone marrow transplantation.

\section{References}

Andrea Bartolini, Ilaria Frasson. 2014. Comparison of phenotypic methods for the detection of carbapenem nonsusceptible Enterobacteriaceae, Gut Pathogens, 6: 13.

Bansal, M., Vyas, N., Sharma, B., Maheshwari, R.K. 2013. Differentiation of Carbapenemase producing Enterobacteriaceae by Triple disc Test. Indian J. Basic Appl. Med. Res., 3(1): 314-320.

Baraniak, J., Fiett, M., Herda, E., et al. 2013. Species distribution, clonal structure and carbapenemase types of carbapenemase- 
producing Entero bacteriaceae in European and Israeli hospitals comparative study. www.congrex.ch/.../2013.ECCMID13

Beth, A., Rasmussen, Karen Bush. 1997. Carbapenem hydrolyzing beta-lacatamses. Antimicrobial Agents Chemother., 41: 221-232.

Binish Arif Sultan, Erum Khan. 2013. Effectiveness of Modified Hodge Test to detect NDM-1 Carbapenemases: an experience from Pakistan; J. Pak. Med. Assoc., Vol. 63, No. 8.

Chauhan, K., Pandey, A. 2015. Evaluation of phenotypic tests for detection of Klebsiella pneumoniae carbapene-mase and metallo-beta-lactamase in clinical isolates of Escherichia coli and Klebsiella species. Indian J. Pathol. Microbiol., 58(1): 31-35.

Clinical and Laboratory Standards Institute CLSI, 2012. Performance Standards for Antimicrobial Susceptibility testing.Twenty second Informational Standard. M100-S22. CLSI, Wayne, PA.

Lee, K., Chong, Y., Shin, H.B., Kim, Y.A., Yong, D., Yum, J.H. 2001. Modified Hodge and EDTA-disk synergy tests to screen metallo- -lactamase-producing strains of Pseudomonas and Acinetobacter species. Clin. Microbiol, Infect., 7: 8891.

Nordmann, P., Cuzon, G., Naas, T. 2009. The real threat of Klebsiella pneumoniae carbapenemase-producing bacteria. Lancet Infect. Dis., 9: 228-36.

Queenan, A.M., Bush, K. $2007 . \quad$ Carbap- enemases: the versatile $\beta$-lactamases. Clin. Microbiol. Rev., 20: 440-58.

Rai, S., Das, D., Niranjan, D.K., Singh, N.P., Kaur, I.R. 2014. Carriage prevalence of carbapenem-resistant Enterobacteria-ceae in stool samples: A surveillance study, Australasian Med. J., 7(2): 64-67.

Sachin Kumar, Seema Bhadauria. 2014. Leading trend of carbapenem resistance in Enterobacteriaceae in India. Int. J. Basic and Appl. Med. Sci., Vol. 4(2): pp. 205208.

Sanjeev Kumar, S.K. Mehra. 2015. Performance of Modified Hodge Test and Combined Disc Test for Detection of Carbapenemases in Clinical Isolates of Enterobacteriaceae Int. J. Curr. Microbiol. App. Sci., 4(5): 255-261.

Sathya Pandurangan, Shamsadh Begum Esak. 2015. Phenotypic Detection Methods of Carbapenemase Production in Enterobacteriaceae. Int. J. Curr. Microbiol. App. Sci., 4(6): 547-552

Tsakris, A., poulou, A., Pournaras, S., Voulgari, E., et al. 2010. A simple phenotypic method for the differentiation of metallo$\beta$-lactamases and class A KPC carbapenemases in Enterobacteriaceae clinical isolates. J. Antimicrob. Chemother., 65: 1664-71.

Wattal, C., Goel, N,. Oberoi, J.K., Raveendran, R., Datta, S., Prasad, K.J. 2010. Surveillance of multidrug resistant organisms in a tertiary care hospital in Delhi, India. J. Assoc. Physicians India, 58: 326.

\section{How to cite this article:}

Swetha, M., K. Anuradha and Kiran Kavatagi. 2016. Phenotypic Detection of Different Resistance Mechanism of Imipenem Resistance among Clinical Isolates of Enterobacteriaceae. Int.J.Curr.Microbiol.App.Sci. 5(7): 234-241. doi: http://dx.doi.org/10.20546/ijcmas.2016.507.024 